\title{
Using a large area CMOS APS for direct chemiluminescence detection in Western Blotting Electrophoresis
}

\author{
Michela Esposito $^{a}$, Jane Newcombe ${ }^{b}$, Thalis Anaxagoras $^{c}$, Nigel M. Allinson ${ }^{c}$ \\ and Kevin Wells ${ }^{a, d}$ \\ ${ }^{a}$ Centre for Vision, Speech and Signal Processing, Faculty of Engineering and Physical \\ Sciences, University of Surrey, Guildford GU2 7XH, UK; \\ ${ }^{b}$ Faculty of Health and Medical Sciences, University of Surrey, Guildford GU2 7XH,UK; \\ ${ }^{c}$ School of Computer Science, University of Lincoln, Lincoln, LN6 7TS, UK; \\ ${ }^{d}$ King Saud University, Riyadh, Saudi Arabia
}

\begin{abstract}
Western blotting electrophoretic sequencing is an analytical technique widely used in Functional Proteomics to detect, recognize and quantify specific labelled proteins in biological samples. A commonly used label for western blotting is Enhanced ChemiLuminescence (ECL) reagents based on fluorescent light emission of Luminol at $425 \mathrm{~nm}$. Film emulsion is the conventional detection medium, but is characterized by non-linear response and limited dynamic range. Several western blotting digital imaging systems have being developed, mainly based on the use of cooled Charge Coupled Devices (CCDs) and single avalanche diodes that address these issues. Even so these systems present key drawbacks, such as a low frame rate and require operation at low temperature. Direct optical detection using Complementary Metal Oxide Semiconductor (CMOS) Active Pixel Sensors (APS)could represent a suitable digital alternative for this application. In this paper the authors demonstrate the viability of direct chemiluminescent light detection in western blotting electrophoresis using a CMOS APS at room temperature. Furthermore, in recent years, improvements in fabrication techniques have made available reliable processes for very large imagers, which can be now scaled up to wafer size, allowing direct contact imaging of full size western blotting samples. We propose using a novel wafer scale APS (12.8 $\mathrm{cm} \times 13.2 \mathrm{~cm}$ ), with an array architecture using two different pixel geometries that can deliver an inherently low noise and high dynamic range image at the same time representing a dramatic improvement with respect to the current western blotting imaging systems.
\end{abstract}

Keywords: western blotting, protein detection, room temperature image sensor, CMOS, APS

\section{INTRODUCTION}

Western Blotting electrophoresis sequencing is an analytical technique widely used in the field of Functional Proteomics to detect, recognize and quantify specific labeled target molecules in biological samples. Fluorescence labelling represents a well established technique to detect biomolecules though it requires complex apparatus comprising an external light source, optics and light filters and requires software procedures to correct background fluorescence. Chemiluminescence labelling represents an alternative to overcome these issues. It requires neither external illumination nor filtering optics and does not produce an inherently label-related background to correct. A suitable choice for chemiluminescence labelling is to use Enhanced Chemiluminescence (ECL) reagents based on light emission at $425 \mathrm{~nm}$ from Luminol (3-amino-phthallhydrazide) cathalized by Horse Radish Peroxidase (HPR). ${ }^{1}$ Film emulsion is the conventional detecting medium in this application though it

Further author information: (Send correspondence to M. Esposito) M. Esposito: E-mail: m.esposito@surrey.ac.uk 

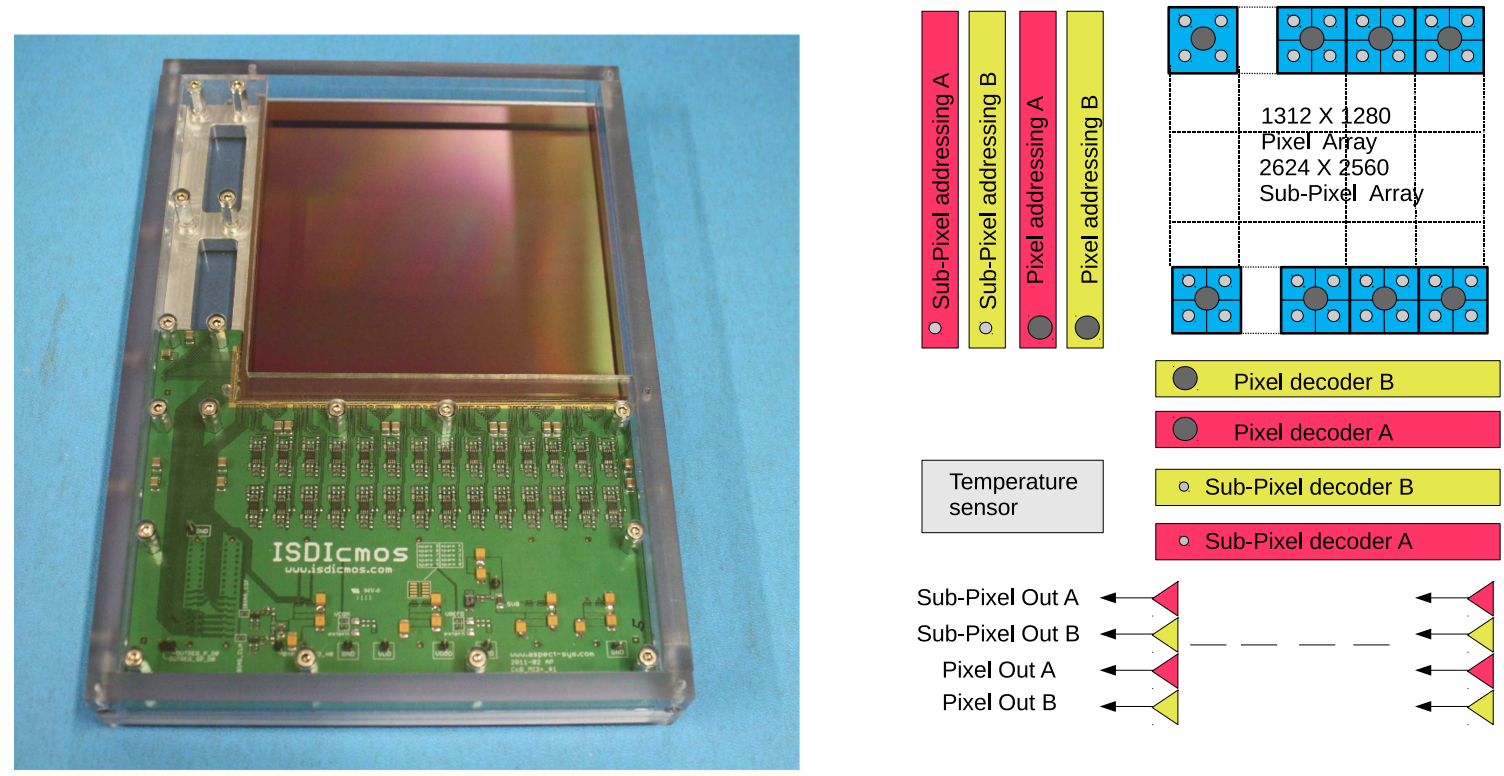

Figure 1. left A picture of the DynAMITe large area sensor in its first deployment. right A schematic of the pixel array arrangement together with a block diagram of the internal architecture of the DynAMITe detector. Yellow circles represent the large size diodes placed at $100 \mu \mathrm{m}$ pitch (Pixels). Cyan circles represent the small size diodes placed at 50 $\mu \mathrm{m}$ pitch (Sub-Pixels).

presents severe limitations such as a limited and non linear dynamic range requiring multiple exposures. It is also a time-consuming and expensive procedure. Several western blotting digital imaging systems have being developed in the last two decades, mainly based on the use of $\mathrm{CCDs}^{2345}$ and single avalanche diodes. ${ }^{6}$ Even so these systems exhibit serious shortcomings in imaging performance, such as a low frame rate due to the inherent sequential read-out, and require operation at low temperature to achieve a reasonable noise level. CMOS Active Pixel Sensors (APS) have gained popularity because of their potential for overcoming such issues, allowing a higher frame rate, due to a column parallel readout together with low power consumption, a low cost technology and the possibility of operations at room temperature. Here, we demonstrate the viability of direct chemiluminescent light detection in western blotting electrophoresis using a CMOS APS at room temperature. Furthermore, in recent years, improvements in design and fabrication techniques have made available manufacturing processes for wafer scale imagers, which can now be seamlessly scaled from a few centimetres square up to the wafer size allowing contact imaging of full size western blotting samples. We propose the use of a new wafer scale APS $(12.8 \mathrm{~cm} \times 13.2 \mathrm{~cm})$ for direct chemiluminescence detection in western blotting. This sensor based on the use of two different diode geometries in the same pixel array and with different size active pixels can deliver an inherently high spatial resolution, low noise and high dynamic range.

\section{MATERIALS AND METHODS}

\subsection{CMOS APS Imaging Detectors}

The detector used is a new large area monolithic APS developed by the Multidimensional Integrated Intelligent Imaging Plus (MI-3 Plus) consortium (EPSRC EP/G037671/1 programme). A picture of the detector in its first 


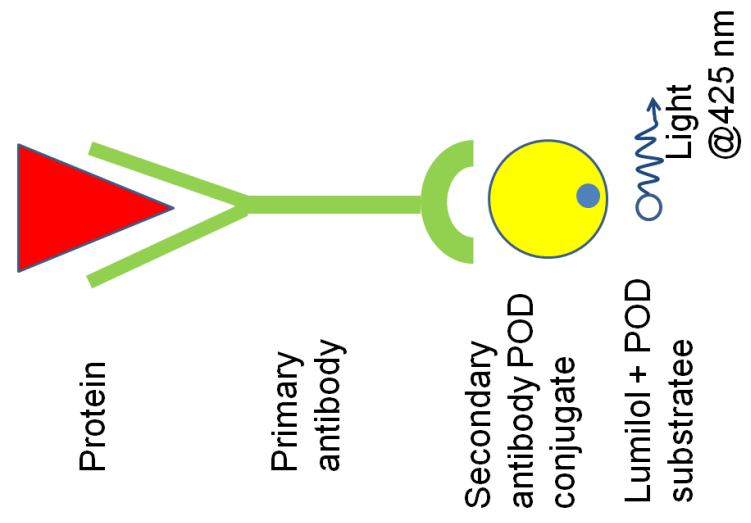

Figure 2. Schematic representation of protein detection in Western Blotting by means of chemiluminescent emission: Proteins bind a specific primary antibody, which in turn binds a specific secondary antibody. The secondary antibody is conjugated with $\mathrm{H}_{2} \mathrm{O}_{2}$ in order to emit light when exposed to a Luminol substrate.

deployment is shown in fig. 1 left. This APS, named the Dynamic Adjustable for Medical Imaging Technology or DynAMITe, was fabricated in a $0.18 \mu \mathrm{m}$ CMOS process using reticule stitching technique to give a total active area of $12.8 \mathrm{~cm} \times 13.2 \mathrm{~cm}$.

The pixel array has been designed to provide two imagers in one by using different size light-converting elements meshed in the same pixel matrix. The detector consists of fine-pitch photodiodes, offering intrinsic low noise and high spatial resolution, and a large-pitch diodes, offering high dynamic range. Both arrays are geometrically superimposed. Thus each cell of the DynAMITe matrix is fitted with multiple diodes: four diodes of small size (50 $\mu \mathrm{m}$ side), named Sub-Pixels, and one diode of large size (100 $\mu \mathrm{m}$ side), named Pixel. The whole matrix comprises $1312 \times 1280$ Pixels and $2624 \times 2560$ Sub-Pixels. Each light-converting element is designed in a standard 3-T architecture, allowing a high Fill Factor (70\%) and Quantum Efficiency (QE), measured as $45 \%$ for $523 \mathrm{~nm}$ light. ${ }^{7}$

Different reset voltages applied to Pixels and Sub-Pixels allow the generation of different depletion widths. Since the Sub-Pixel diodes are reset at a higher voltage, this camera rapidly collects the initially generated photoelectrons which ensures an intrinsic lower noise and a higher spatial resolution. Then, after the Sub-Pixels reach near saturation, the Pixel diodes start collecting so offering a higher dynamic range due to the larger collecting area compared to the Sub-Pixels. With these design features the DynAMITe detector is an imager capable of simultaneous low noise and high resolution, due to the Sub-Pixels, and a high dynamic range with a relatively low noise due to the Pixels.

Four independent read out circuits were realized for this sensor leading to a significant increase in the frame rate up to $30 \mathrm{fps}$ for the Sub-Pixel array and $94 \mathrm{fps}$ for the Pixel array.

Both the Pixel and Sub-Pixel matrices can be read in combination, or separately with different exposure times, in destructive or non-destructive mode (see fig. 1 right). Furthermore groups of regions of Sub-Pixels can be read out selectively for on-line evaluation of the source activity and the required exposure time.

\subsection{Biological samples and experimental set-up}

Protein detection in western blotting is a multi-step process schematically represented in Fig. 2 In fact in order to be detected, proteins need to be chemically bound in several stages until the last stage where light is emitted for detection. Proteins bind a specific primary antibody, which then binds a secondary specific antibody.The 


\begin{tabular}{|ccccccccccccc|}
\hline & 1 & 2 & 3 & 4 & 5 & 6 & 7 & 8 & 9 & 10 & 11 & 12 \\
A & 9 & 10 & 11 & 12 & 13 & 14 & 15 & 16 & 17 & 18 & 19 & 20 \\
B & 1.2 & 1.4 & 1.6 & 1.8 & 2 & 1 & 2 & 3 & 4 & 5 & 6 & 7 \\
C & 0 & 0.1 & 0.12 & 0.14 & 0.16 & 0.18 & 0.2 & 0.1 & 0.2 & 0.4 & 0.6 & 0.7 \\
\hline
\end{tabular}

Figure 3. A map distribution of the sample concentration. Concentration of anti-human secondary antibody (top) and E. Coli proteins Values are expressed in $\mathrm{nl}$.

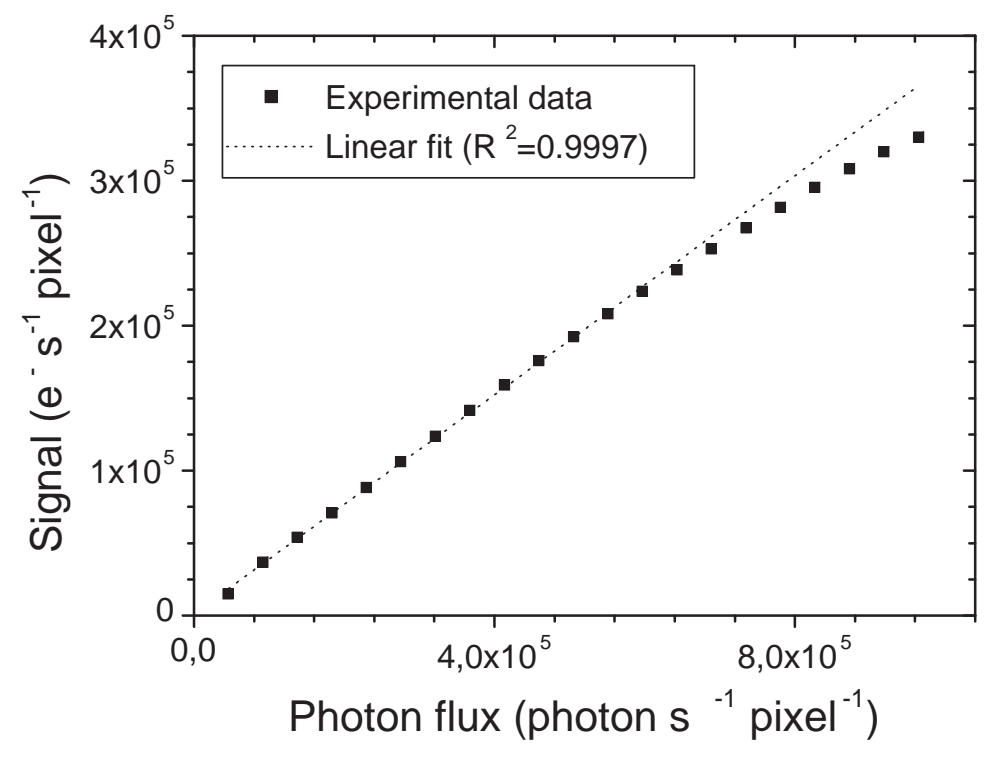

Figure 4. The detector response curve to blue light: the specific signal in a ROI is plotted versus light source irradiance. A linear fit is shown as a dotted line. 
secondary antibodies are bound to $\mathrm{H}_{2} \mathrm{O}_{2}$ molecules in order to emit light when exposed to a Luminol substrate, according to the following chemical reaction:

$$
\text { Luminol } \rightarrow 3-A P A[\diamond] \rightarrow 3-A P A+\gamma(425 n m)
$$

A $425 \mathrm{~nm}$ photon is emitted as a product of this reaction.

The last stage of the detection chain has been investigated in this work, in order to evaluate the response of this CMOS system to chemiluminescence. Secondary anti-human IgG HRP antibody (A8667, Sigma-Aldrich), ranging from ranging 20 to $0.1 \mathrm{nl}$ diluted in Tris Buffered Saline, have been spotted on a Hybond - LEP membrane (Amersham Biosciences) in a dot blot manifold. Secondary antibody concentration is reported in fig. 3. The membrane was then washed in a Lumi-Light Western Blotting Substrate (Roche Applied Science) in order to activated the ECL emission by means of the Luminol contained. The sample was prepared in duplicate to be exposed to the DynAMITe digital detector and film emulsion. The sample was placed in close contact with the detector surface and imaged in a light-tight box, and also to film emulsion. Images were acquired at 1-second frame rate and dark corrected. The samples was also exposed to film emulsion in a light-tight box in dark room. Standard procedures for film development were adopted. Furthermore the Sub-Pixel camera of the DynAMITe detector has been tested in this work with a $468 \mathrm{~nm}$ Light Emitting Diode (LED) array - close to that produced by chemiluminescence $(425 \mathrm{~nm})$ - in order to asses the detector response.

\section{RESULTS}

\subsection{Linearity and Quantum Efficiency}

Figure 4 shows the linearity plot for the DynAMITe detector exposed to blue light. The specific detected signal in a ROI per time and area unit, after signal calibration ${ }^{7}$ is plotted versus the source light irradiance. A linear fit has been performed for this data and is shown as a dotted line in figure 4. The DynAMITe detector presents a linear response to blue light in the range 0.1-7.5 $\times 10^{5}$ photons $\mathrm{s}^{-1} \mathrm{~mm}^{-2}$ and a sensitivity of $(0.38 \pm 0.1)$ $\mathrm{e}^{-} /$photon, calculated as the slope of the linear region in the response curve, corresponding to a quantum efficiency of $38 \%$ at $468 \mathrm{~nm}$.

\subsection{Chemiluminescence detection}

Membranes, spotted with secondary antibody activated with Luminol, were exposed to the DynAMITe detector and film emulsion. Figure 5 shows a comparison between film emulsion (left column) and DynAMITe detector (right column) in chemiluminescence detection. The reference to the dot position is reported for all the images according to the distribution map of fig. 3. Images are reported for 1-second (fig. $5 a, f$ ), 3 -second (fig. $5 a, f$ ) 5 -second (fig. $5 c, h$ ), 30-second (fig. $5 d, i$ ) and 1 minute (fig. $5 e, j$ ) exposure time.

Lowest activity blots in fig. 5 (C2-C10) are not visible in the film image with 1 second exposure time (fig. 5 a). On the other side the same blots are clearly visible above the background with the same exposure time for the DynAMITe detector (fig. $5 \mathrm{f}$ ), thus resulting in an higher sensitivity of the digital detector compared to film. An horizontal profile of row $\mathrm{C}$ for row $\mathrm{C}$ imaged exposed for 1 second to film emulsion (left) and to DynAMITe is reported in fig. 6. In 1-second film image only the two highest activity blots (C12 and C11) are distinguishable as peaks above the background $(6(l e f t))$, whereas all the spotted blots are distinguishable in $(6$ (left)).

Film images at $5 \mathrm{~s}, 30 \mathrm{~s}$ and 1 min (fig. 5 b-e) show saturation for all the blots in rows A-B. This leads to a spread of the signal on the film resulting in the incapability of distinguish different blots. On the other side saturation is absent in all the images reported for DynAMITe (fig. $5 f-j$ ) because of a larger dynamic range. 


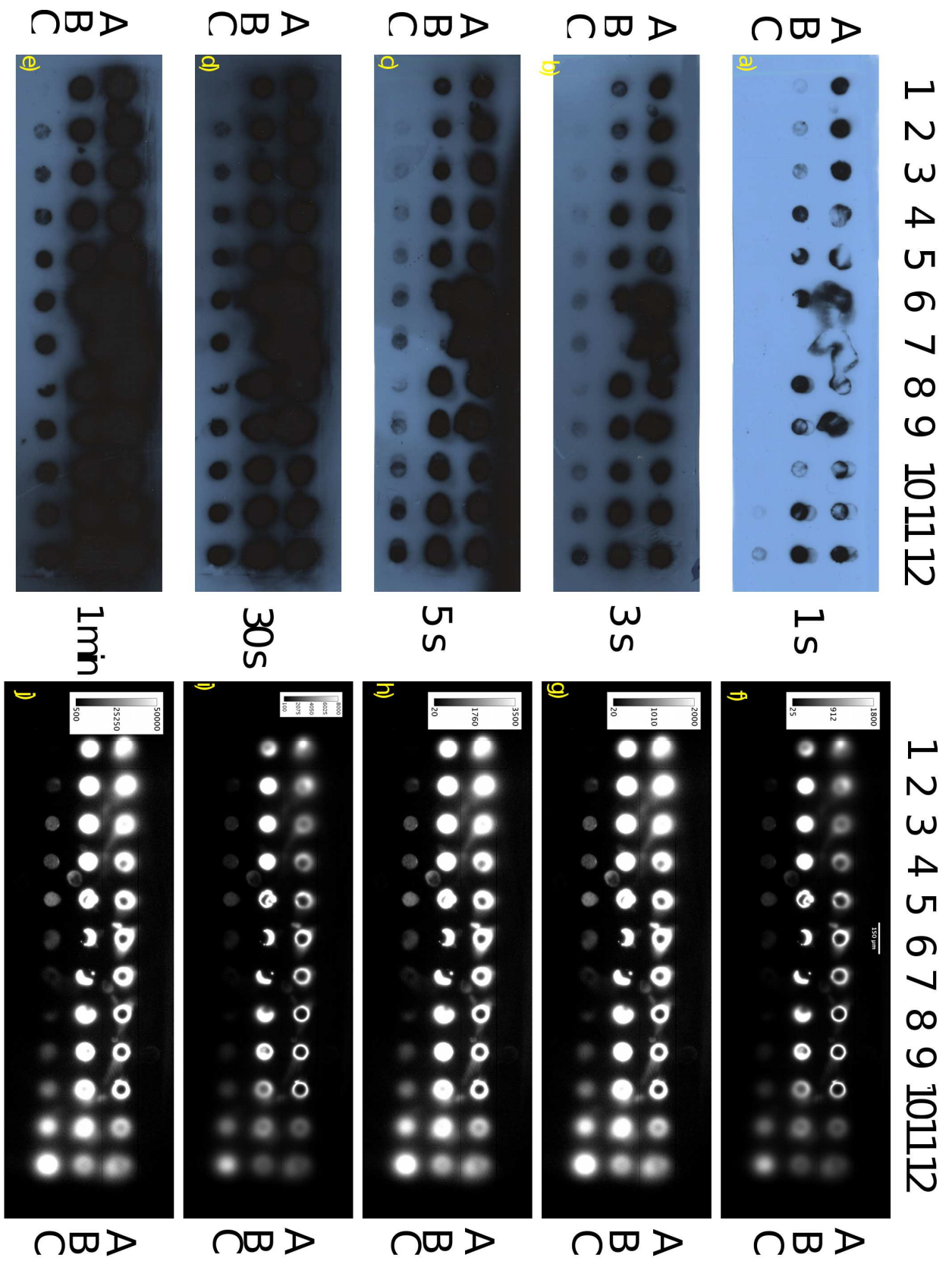

Figure 5. Secondary anti-human antibody activated with Luminol imaged with film emulsion (left) and with the DynAMITe detector (right). Images taken at several exposure times are reported: $1 \mathrm{~s}(a, f), 3 \mathrm{~s}(b, g) 5 \mathrm{~s}(c, h), 30 \mathrm{~s}(d, i)$ and $1 \mathrm{~min}(e, j)$. The reference to the dot position is reported for all the images according to the distribution map of fig. 3 . 

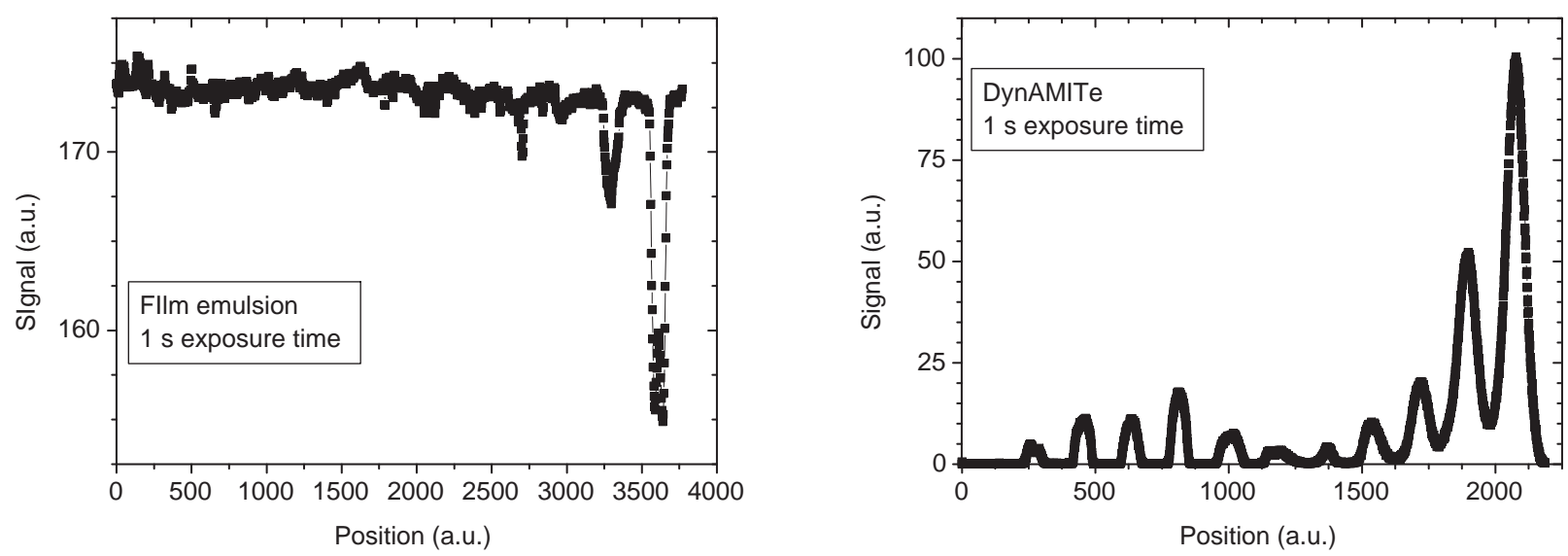

Figure 6. Horizontal profile of blots of row C imaged with film emulsion left and the DynAMITe detector right for 1 second.

\section{CONCLUSIONS}

The DynAMITe detector has been presented as a novel large area APS detector capable of two inherently different resolutions each with different noise and saturation performance in the same pixel array. The viability of this APS for direct chemiluminescence detection in western blotting has been demonstrated. The detector response to chemiluminescence light has been tested and a quantum efficiency of $38 \%$ has been measured. Performance comparison with film images shows a larger dynamic range, a higher sensitivity and a lower minimum detectable signal for this detector. Its wide dynamic range, due to an inherently dual resolution, has been proven to overcome prompt saturation and necessity of multiple exposures, common drawbacks in this field.

\section{ACKNOWLEDGMENTS}

This work was supported by the EPSRC Multidimensional Integrated Intelligent Imaging Plus (MI-3 Plus) programme (EP/G037671/1)

\section{REFERENCES}

[1] Thorpe, G. et al., "Enhanced chemiluminescence reactions catalyzed by horseradish peroxidase," Meth. Enzymol. 133, 331-354 (1986).

[2] Mahon, A. et al., "A ccd based system for the detection of dna in electrophoresis gel by uv absorption," Phys. Med. Biol. 44, 1529-41 (1999).

[3] [FluorChemQ System, Quantitative Western Blot imaging], Cell Biosciences (online). http://www.cellbiosciences.com/fluorchemq.html.

[4] [Ettan DIGE], GE Healthcare (online). http://gelifesciences.com.

[5] [Chemidoc XRS + System], BioRad Laboratories (online). http://www.bio-rad.com.

[6] Gersbach, M., "A room temperature cmos single photon sensor for cheiluminescence detection," presented at Internation Conference on Miniaturized Systems for Chemistry and Life Science (MicroTas) (November 2006). 
[7] Esposito, M. et al., "Dynamite: a wafer scale sensor for biomedical applications," Journ. of Instr. 6, C12064 (2011). 